\title{
ON THE NON-EXISTENCE OF A CYCLIC HOMOLOGY THEORY WITH COEFFICIENTS
}

\author{
G. Donadze And M. LADRA
}

\begin{abstract}
We show that one cannot construct a cyclic homology theory with coefficients that would be related to the Hochschild homology by the Connes periodicity exact sequence. We show that this is impossible even if the ideals of a given algebra have been taken as coefficients. Despite this, the cyclic homology with coefficients can be defined by restricting the class of coefficient modules. In particular, if $\gamma: I \rightarrow A$ is a crossed module of algebras with $I$ being $H$-unital, then it is possible to define "nice" cyclic homology groups of $A$ with coefficients in $I$.
\end{abstract}

\section{Formulation of a problem}

Let $\mathbf{k}$ be a commutative unital ring and $A$ be an associative unital algebra over $\mathbf{k}$. For any bimodule $M$ over $A$ we have a non-negatively graded complex, called the Hochschild complex,

$$
C_{*}(A, M):=\cdots \stackrel{b}{\longrightarrow} M \otimes A^{\otimes 2} \stackrel{b}{\longrightarrow} M \otimes A \stackrel{b}{\longrightarrow} M,
$$

where $b$ is the Hochschild boundary and $\otimes=\otimes_{\mathbf{k}}$. The homology groups of $C_{*}(A, M)$ define the Hochschild homologies of $A$ with coefficients in $M$, denoted by $H_{n}(A, M)$ in general and by $H H_{n}(A)$, when $M=A$, where $n \geq 0$.

Let $H C_{n}(A), n \geq 0$, denote the $n$th cyclic homology group of $A$ (see [5] for the definition). The following exact sequence is well known

$$
\cdots \stackrel{\delta_{n-1}}{\longrightarrow} H H_{n}(A) \stackrel{i_{n}}{\longrightarrow} H C_{n}(A) \stackrel{s_{n}}{\longrightarrow} H C_{n-2}(A) \stackrel{\delta_{n-2}}{\longrightarrow} H H_{n-1}(A) \stackrel{i_{n-1}}{\longrightarrow} \cdots
$$

and is called the Connes periodicity exact sequence (see $[1,5]$ ). Denote by ${ }_{A} \mathcal{M}_{A}$ the category of bimodules over $A$ and by Mod the category of k-modules. Then, according to the aforementioned we have the functors $H_{n}(A,-):{ }_{A} \mathcal{M}_{A} \longrightarrow \operatorname{Mod}, n \geq 0$.

Question 1.1. Is it possible to construct functors $H C_{n}(A,-):{ }_{A} \mathcal{M}_{A} \longrightarrow \operatorname{Mod}, n \geq$ 0 , satisfying the following conditions?

(Q1) there exist natural transformations

$$
\begin{aligned}
\mu_{n}: H_{n}(A,-) \longrightarrow H C_{n}(A,-), & \nu_{n}: H C_{n}(A,-) \longrightarrow H C_{n-2}(A,-) \text { and } \\
& \lambda_{n}: H C_{n}(A,-) \longrightarrow H_{n+1}(A,-), \\
\text { such that } \mu_{n}(A)=i_{n}, \nu_{n}(A)= & s_{n}, \lambda_{n}(A)=\delta_{n} ;
\end{aligned}
$$

Received by the editors January 3, 2012.

1991 Mathematics Subject Classification. 18G10, 18G50, 18G60, 16E40.

Key words and phrases. Hochschild and cyclic homology, H-unital algebra, crossed modules of algebras.

Dedicated to the memory of Professor J.-L. Loday. 
(Q2) the following sequence is exact:

$\cdots \stackrel{\lambda_{n-1}}{\longrightarrow} H_{n}(A,-) \stackrel{\mu_{n}}{\longrightarrow} H C_{n}(A,-) \stackrel{\nu_{n}}{\longrightarrow} H C_{n-2}(A,-) \stackrel{\lambda_{n-2}}{\longrightarrow} H_{n-1}(A,-) \stackrel{\mu_{n-1}}{\longrightarrow} \cdots$.

In particular, Question 1.1 asks whether or not there exists cyclic homology theory with coefficients. In the very interesting paper [4], this question is studied in a different context. The answer to this question is negative in general (see Section 2), but if one specifies the class of bimodules, then one can define the functors satisfying (Q1) and (Q2). In particular, if an algebra $A$ is fixed, then for any crossed module of algebras $\gamma: I \rightarrow A$ with $I$ being $H$-unital, one can define the cyclic homology groups $H C_{*}(A, I)$ satisfying our requirement (see Proposition 2.7).

\section{Non existence of the cyclic homology theory with coefficients}

We show that if $A$ is a polynomial algebra in $n$ variables, $n \geq 1$, then Question 1.1 has negative answer.

Lemma 2.1. Let $A=\mathbf{k}\left[x_{1}, \ldots, x_{n}\right], n \geq 1$. Then there is no natural transformation $\eta: H_{0}(A,-) \rightarrow H_{1}(A,-)$ such that $\eta(A)=\delta_{0} i_{0}$, where $i_{0}: H H_{0}(A) \rightarrow H C_{0}(A)$ and $\delta_{0}: H C_{0}(A) \rightarrow H H_{1}(A)$ are defined from the Connes periodicity exact sequence.

Proof. To prove the lemma, assume the contrary. Then there exists a natural transformation $\eta: H_{0}(A,-) \rightarrow H_{1}(A,-)$ such that $\eta(A)=\delta_{0} i_{0}$. Let $I$ be an ideal of $A$ generated by $x_{1}, \ldots, x_{n}$. Denote by $\sigma$ the natural inclusion $I \subset A$. Then, we have the following commutative diagram:

$$
\begin{array}{cc}
H_{0}(A, I) \stackrel{\eta(I)}{\longrightarrow} H_{1}(A, I) \\
H_{0}(A, \sigma) \downarrow \\
H_{1}(A, \sigma) \downarrow \\
H_{0}(A, A) \stackrel{\eta(A)}{\longrightarrow} H_{1}(A, A) .
\end{array}
$$

Hence, we have the following commutative diagram:

$$
\begin{aligned}
I & \stackrel{\eta(I)}{\longrightarrow} H_{1}(A, I) \\
\sigma & H_{1}(A, \sigma) \downarrow \\
& \downarrow \\
A & \stackrel{\eta(A)}{\longrightarrow} H_{1}(A, A) .
\end{aligned}
$$

This implies that

$$
(\eta(A) \circ \sigma)\left(x_{1}\right)=\left(H_{1}(A, \sigma) \circ \eta(I)\right)\left(x_{1}\right) .
$$

Since $\eta(A)=\delta_{0} i_{0},(\eta(A) \circ \sigma)\left(x_{1}\right)=\overline{1 \otimes x_{1}}$, where $\overline{1 \otimes x_{1}}$ denotes the natural image of $1 \otimes x_{1}$ in $H_{1}(A, A)$. On the other hand,

$$
\operatorname{Im}\left(H_{1}(A, \sigma) \circ \eta(I)\right) \subset \operatorname{Im}\left(H_{1}(A, \sigma)\right)
$$

implies that

$$
\overline{1 \otimes x_{1}} \in \operatorname{Im}\left(H_{1}(A, \sigma)\right) .
$$

Observe that $\operatorname{Im}\left(H_{1}(A, \sigma)\right)$ is a submodule of $H_{1}(A, A)$ generated by all $\overline{c \otimes a}$, where $c \in I, a \in A$ and $\overline{c \otimes a}$ is the natural image of $c \otimes a$ in $H_{1}(A, A)$. Hence, there are 
$c_{1}, \ldots, c_{m} \in I$ and $a_{1}, \ldots, a_{m} \in A$ such that

$$
\overline{1 \otimes x_{1}}=\sum_{i=1}^{m} \overline{c_{i} \otimes a_{i}} .
$$

Taking into account the isomorphism between $H_{1}(A, A)$ and $\Omega_{A / k}^{1}$, we obtain:

$$
d x_{1}=\sum_{i=1}^{m} c_{i} d a_{i} .
$$

Moreover, we have

$$
d a_{i}=\sum_{j=1}^{n} b_{i j} d x_{j}
$$

where $b_{i j} \in A$. Combining the last two equalities, we get:

$$
d x_{1}=\sum_{i=1}^{m} \sum_{j=1}^{n} c_{i} b_{i j} d x_{j}
$$

This is a contradiction, since $c_{i} b_{i j} \in I$ and $\Omega_{A / k}^{1}$ is a free $A$-module with basis $d x_{1}, \ldots, d x_{n}$.

Corollary 2.2. Let $A=\mathbf{k}\left[x_{1}, \ldots, x_{n}\right], n \geq 1$. Then there are no functors satisfying (Q1).

Proof. Assume the contrary and define a natural transformation $\eta: H_{0}(A,-) \rightarrow$ $H_{1}(A,-)$ in the following way: $\eta(M)=\lambda_{0}(M) \mu_{0}(M)$ for all $M \in{ }_{A} \mathcal{M}_{A}$. So $\eta(A)=$ $\lambda_{0}(A) \mu_{0}(A)=i_{0} \delta_{0}$. But this is a contradiction by Lemma 2.1.

Corollary 2.2 implies that for $A=\mathbf{k}\left[x_{1}, \ldots, x_{n}\right], n \geq 1$, Question 1.1 has negative answer. It is even impossible to construct a functor $H C_{0}(A,-):{ }_{A} \mathcal{M}_{A} \longrightarrow$ Mod which admits property (Q1).

Example 2.3. Let $A=\mathbf{k}$. Set $H C_{0}(\mathbf{k}, M)=M$ and $H C_{n}(\mathbf{k}, M)=0$, when $n \geq 1$ for all $M \in{ }_{A} \mathcal{M}_{A}$. Then, the functors $H C_{n}(\mathbf{k},-):{ }_{A} \mathcal{M}_{A} \longrightarrow \operatorname{Mod}, n \geq 0$, satisfy (Q1) and (Q2).

This trivial example is the only one (known to us) for which Question 1.1 has positive answer. One might ask what happens if in Question 1.1 the source category ${ }_{A} \mathcal{M}_{A}$ is replaced by a specific class of bimodules over $A$. We will consider a few examples regarding this.

Define a category $\mathcal{A}$ in the following way: $I \in \operatorname{ob}(\mathcal{A})$ if $I$ is an ideal of $A$, and for any $I, I^{\prime} \in \operatorname{ob}(\mathcal{A}), f: I \rightarrow I^{\prime}$ is a morphism in $\mathcal{A}$ if $f$ is k-linear map satisfying the following conditions: $f(a x)=a f(x)$ and $f(x a)=f(x) a$, for all $a \in A, x \in I$. Then using the same arguments as above one can show that for $A=\mathbf{k}\left[x_{1}, \ldots, x_{n}\right], n \geq 1$, there are no functors $H C_{m}(A,-): \mathcal{A} \rightarrow \operatorname{Mod}, m \geq 0$, satisfying (Q1) and (Q2). This means that we are not able to define cyclic homology groups of $A$ even if the ideals have been taken as coefficients.

Let $\mathcal{A}^{\prime}$ be the full subcategory of $\mathcal{A}$ consisting of all $H$-unital ideals of $A$. We remind the reader that if $\mathbf{k}$ is a field, then an algebra $I$ is $H$-unital (see [5]; cf. [6] for the 
definition of $H$-unital algebras in the case commutative unital ring) iff the homology groups of the bar complex $C_{*}^{\text {bar }}(I)$ are trivial, where

$$
C_{*}^{\text {bar }}(I): \cdots \rightarrow I^{\otimes n+1} \stackrel{b^{\prime}}{\longrightarrow} I^{\otimes n} \stackrel{b^{\prime}}{\longrightarrow} \cdots \stackrel{b^{\prime}}{\longrightarrow} I^{\otimes 2} \stackrel{b^{\prime}}{\longrightarrow} I,
$$

and the differential $b^{\prime}: I^{\otimes n+1} \rightarrow I^{\otimes n}$ is given by the formula

$$
b^{\prime}\left(x_{0}, x_{1}, \ldots, x_{n}\right):=\sum_{i=0}^{n-1}(-1)^{i}\left(x_{0}, x_{1}, \ldots, x_{i} x_{i+1}, \ldots, x_{n}\right) .
$$

Proposition 2.4. Let $\mathbf{k}$ be a field and $A$ be a unital algebra over $\mathbf{k}$. Then there are functors $H C_{n}(A,-): \mathcal{A}^{\prime} \longrightarrow$ Mod, $n \geq 0$, satisfying (Q1) and (Q2).

Proof. Since the Hochschild and cyclic homologies are defined for non unital algebras (see [5]), we can set $H C_{n}(A, I)=H C_{n}(I), n \geq 0$, for any $I \in \mathcal{A}^{\prime}$. Moreover, by [2] we have a natural isomorphism $H_{n}(A, I)=H H_{n}(I)$, for all $H$-unital ideal $I \subset A$. Now the Connes periodicity exact sequence written for $I$,

$$
\cdots \rightarrow H H_{n}(I) \rightarrow H C_{n}(I) \rightarrow H C_{n-2}(I) \rightarrow H H_{n-1}(I) \rightarrow \cdots,
$$

gives the desired result.

Thus we have proved that one can construct a cyclic homology theory of an algebra $A$ with coefficients in $H$-unital ideals. This is not as strong a result as one would desire, since $H$-unital ideals form a very restrictive class on the one hand, and on the other hand, $H C_{*}(A, I)$ is nothing but $H C_{*}(I)$, by Proposition 2.4. In the rest of the paper, we prove that we can construct a cyclic homology theory for a slightly wider class of bimodules over $A$. For this we need to recall the notion of a crossed module of algebras introduced by Ellis [3].

Definition 2.5. Let $I$ and $A$ be not necessarily unital algebras. Then, an algebra homomorphism $\gamma: I \rightarrow A$ is called a crossed module of algebras, if $I$ is equipped with bimodule structure over $A$ and the following conditions hold:

$$
\begin{aligned}
& \gamma(a x)=a \gamma(x), \quad \gamma(x a)=\gamma(x) a, \\
& \gamma(x) x^{\prime}=x x^{\prime}=x \gamma\left(x^{\prime}\right) \quad \text { (Peiffer Identity), }
\end{aligned}
$$

for all $a \in A, x, x^{\prime} \in I$.

Let $\gamma: I \rightarrow A$ be a crossed module of algebras. Define a homomorphism of complexes $\gamma_{*}: C_{*}(I, I) \rightarrow C_{*}(A, I)$ :

$$
\gamma_{n}\left(x_{0}, x_{1}, \ldots, x_{n}\right)=\left(x_{0}, \gamma\left(x_{1}\right), \ldots, \gamma\left(x_{n}\right)\right), n \geq 0
$$

for all $x_{0}, \ldots, x_{n} \in I$.

Proposition 2.6. Let $\mathbf{k}$ be a field and $I$ be an $H$-unital algebra over $\mathbf{k}$. Then $\gamma_{*}$ is a quasi-isomorphism for any crossed module of algebras $\gamma: I \rightarrow A$.

We will prove this proposition in the next section. As a result, we obtain that if $\mathbf{k}$ is a field and $A$ is unital over $\mathbf{k}$, then there is a natural isomorphism $H_{n}(A, I)=H H_{n}(I)$, $n \geq 0$, for any crossed module of algebras $\gamma: I \rightarrow A$ with $I$ being $H$-unital.

Define a category $\mathcal{B}$ in the following way: $I \in \mathrm{ob}(\mathcal{B})$ if there is a crossed module of algebras $\gamma: I \rightarrow A$. Moreover, for any two crossed module of algebras $\gamma: I \rightarrow A$ and 
$\gamma^{\prime}: I^{\prime} \rightarrow A$, a k-linear map $f: I \rightarrow I^{\prime}$ is a morphism in $\mathcal{B}$ if it preserves the bimodule structure over $A$ and the following diagram is commutative:

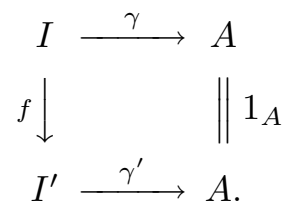

Denote by $\mathcal{B}^{\prime}$ the full subcategory of $\mathcal{B}$ consisting of all $I \in \mathrm{ob}(\mathcal{B})$ such that $I$ is an $H$-unital algebra.

Proposition 2.7. Let $\mathbf{k}$ be a field and $A$ be a unital algebra over $\mathbf{k}$. Then there are functors $H C_{n}(A,-): \mathcal{B}^{\prime} \longrightarrow$ Mod, $n \geq 0$, satisfying (Q1) and (Q2).

Proof. For any $I \in \operatorname{ob}(\mathcal{B})$, set $H C_{n}(A, I)=H C_{n}(I), n \geq 0$. By Proposition 2.6, we can use the same arguments as in Proposition 2.4.

We end this section by giving examples of crossed modules of algebras $\gamma: I \rightarrow A$, where $I$ is $H$-unital but not an ideal in $A$.

Example 2.8. Let $\mathbf{k}$ be a field and $l \geq 2$, where $l$ is an integer. Denote by $J$ the augmentation ideal of the algebra $\mathbf{k}\left[t, t^{1 / l}, t^{1 / l^{2}}, \ldots\right]$. In other words, $J$ is the augmentation ideal of the semigroup algebra $\mathbf{k}[T]$, where $T$ is the additive semigroup generated by $1,1 / l, 1 / l^{2}, \ldots$ Set $I=J / t J$. In [6], it is proved that $I$ is an $H$-unital algebra with non trivial annihilator, in particular $\operatorname{Ann}(I)=(t \mathbf{k}[t]+t J) / t J$. Set $A=$ $\mathbf{k} \oplus(I / \operatorname{Ann}(I))$ and define multiplication in $A$ by $\left(k_{1}, x_{1}\right)\left(k_{2}, x_{2}\right)=\left(k_{1} k_{2}, k_{1} x_{2}+\right.$ $\left.k_{2} x_{1}+x_{1} x_{2}\right)$ for each $k_{1}, k_{2} \in \mathbf{k}, x_{1}, x_{2} \in I / \operatorname{Ann}(I)$. Then $A$ is unital and the natural homomorphism $\gamma: I \rightarrow A$, given by $x \mapsto(0, x)$, is a crossed module of algebras. It is clear that $\gamma$ is not injective, since $\operatorname{Ker} \gamma=\operatorname{Ann}(I) \neq 0$. Furthermore, it can be checked that no ideal in $A$ is isomorphic to $I$.

Example 2.9. Let $\gamma: I \rightarrow A$ be defined as in Example 2.8. Then the natural map $I^{\times n} \rightarrow A^{\times n}, n \geq 1$, is a crossed module of algebras, where $I^{\times n}$ is $H$-unital algebra and no ideal in $\bar{A}^{\times n}$ is isomorphic to $I^{\times n}$.

Example 2.10. Let $\gamma: I \rightarrow A$ be defined as in Example 2.8. Then the natural map $M_{n}(I) \rightarrow M_{n}(A)$ is a crossed module of algebras, where $M_{n}(I)$ and $M_{n}(A)$ denote the ring of $n \times n$ matrices over $I$ and $A$. Moreover, $M_{n}(I)$ is $H$-unital algebra and no ideal in $M_{n}(A)$ is isomorphic to $M_{n}(I)$.

\section{Proof of Proposition 2.6}

In this section, $\mathbf{k}$ is supposed to be a field.

Let $I$ be an algebra. For any submodule $N$,

$$
N \subset \operatorname{Ann}(I)=\{x \in I \mid x y=0=y x, \text { for all } y \in I\},
$$

we have a crossed module of algebras $\tau: I \rightarrow I / N$ defined by natural projection. The following lemma is a special case of Proposition 2.6.

Lemma 3.1. If $I$ is an $H$-unital algebra, then $\tau_{*}: C_{*}(I, I) \rightarrow C_{*}(I / N, I)$ is a quasiisomorphism. 
Proof. Since $\tau_{*}$ is onto, it suffices to show that $\operatorname{Ker} \tau_{*}$ is acyclic. Clearly, $\operatorname{Ker} \tau_{0}=0$ and for $n \geq 1$,

Ker $\tau_{n}=$ linear span of $\left\{x_{0} \otimes x_{1} \otimes \cdots \otimes x_{n} \in I^{\otimes n+1} \mid\right.$ at least one $\left.x_{i} \in N, 1 \leq i \leq n\right\}$.

Consider the following filtration of $\operatorname{Ker} \tau_{n}: F_{0}\left(\operatorname{Ker} \tau_{n}\right)=0, F_{p}\left(\operatorname{Ker} \tau_{n}\right)=\operatorname{Ker} \tau_{n}$ when $p \geq n$ and

$$
\begin{gathered}
F_{p}\left(\operatorname{Ker} \tau_{n}\right)=\text { linear span of }\left\{x_{0} \otimes x_{1} \otimes \cdots \otimes x_{n} \in I^{\otimes n+1} \mid \exists i, i \in\{n-p+1, \ldots, n\}\right. \\
\text { such that } \left.x_{i} \in N\right\},
\end{gathered}
$$

when $p \in\{1, \ldots, n\}$. This defines a filtration of the complex $\operatorname{Ker} \tau_{*}$. We show that the spectral sequence induced by this filtration is trivial at first level, i.e., the homology groups of the following complex

$$
\cdots \stackrel{b}{\longleftarrow} F_{p}\left(\operatorname{Ker} \tau_{n-1}\right) / F_{p-1}\left(\operatorname{Ker} \tau_{n-1}\right) \stackrel{b}{\longleftarrow} F_{p}\left(\operatorname{Ker} \tau_{n}\right) / F_{p-1}\left(\operatorname{Ker} \tau_{n}\right) \stackrel{b}{ } \cdots
$$

are trivial for all $p \geq 1$.

Let $X \subset N$ be a basis of $N$ over $\mathbf{k}$ and $Y \subset I \backslash N$ be a basis of $I / N$ over $\mathbf{k}$. Assume that $w \in F_{p}\left(\operatorname{Ker} \tau_{n}\right)$ and $b(w) \in F_{p-1}\left(\operatorname{Ker} \tau_{n-1}\right)$ for $n \geq 1$ and $p \in\{1, \ldots, n\}$. Then,

$$
w=\sum_{j=1}^{m} w_{0 j} \otimes x_{n-p+1} \otimes x_{n-p+2 j} \otimes \cdots \otimes x_{n j}+w^{\prime},
$$

where $w^{\prime} \in F_{p-1}\left(\operatorname{Ker} \tau_{n}\right), w_{0 j} \in I^{\otimes n-p+1}, x_{n-p+1} \in X, x_{i j} \in Y$ when $i>n-p+1$ and

$$
\left(x_{n-p+1 j}, x_{n-p+2}, \ldots, x_{n j}\right) \neq\left(x_{n-p+1 j^{\prime}}, x_{n-p+2 j^{\prime}}, \ldots, x_{n j^{\prime}}\right), \quad j \neq j^{\prime} .
$$

Since $N \subset \operatorname{Ann}(I)$, we obtain:

$$
b(w)=\sum_{j=1}^{m} b^{\prime}\left(w_{0 j}\right) \otimes x_{n-p+1 j} \otimes x_{n-p+2 j} \otimes \cdots \otimes x_{n j}+w^{\prime \prime},
$$

where $b^{\prime}$ is the differential of the bar complex $C_{*}^{\text {bar }}(I)$ and $w^{\prime \prime} \in F_{p-1}\left(\operatorname{Ker} \tau_{n-1}\right)$. Since $b(w) \in F_{p-1}\left(\operatorname{Ker} \tau_{n-1}\right)$, it follows that $b^{\prime}\left(w_{0 j}\right)=0$ for all $j \in\{1, \ldots, m\}$. Hence there exists $v_{j} \in I^{\otimes n-p+2}$ such that $b^{\prime}\left(v_{j}\right)=w_{0 j}$. Set

$$
\bar{w}=\sum_{j=1}^{m} v_{j} \otimes x_{n-p+1} \otimes x_{n-p+2 j} \otimes \cdots \otimes x_{n j} .
$$

Observe that $\bar{w} \in F_{p}\left(\operatorname{Ker} \tau_{n+1}\right)$ and $b(\bar{w}) \in w+F_{p-1}\left(\operatorname{Ker} \tau_{n}\right)$, which implies that every cycle of the aforementioned complex is a boundary.

Definition 3.2. Let $C_{*}^{\mathrm{bar}}(I / N, I)$ be a non negatively graded complex defined in the following way:

$$
C_{*}^{\text {bar }}(I / N, I):=\cdots \stackrel{b^{\prime}}{\longrightarrow} I \otimes(I / N)^{\otimes 2} \stackrel{b^{\prime}}{\longrightarrow} I \otimes(I / N) \stackrel{b^{\prime}}{\longrightarrow} I,
$$

where

$$
b^{\prime}\left(x, y_{1}, \ldots, y_{n}\right):=\left(x y_{1}, y_{2}, \ldots, y_{n}\right)+\sum_{i=1}^{n-1}(-1)^{i}\left(x, y_{1}, \ldots, y_{i} y_{i+1}, \ldots, y_{n}\right)
$$


for all $x \in I, y_{1}, \ldots, y_{n} \in I / N$. Define a homomorphism of complexes $\tau_{*}^{\prime}: C_{*}^{\text {bar }}(I) \rightarrow$ $C_{*}^{\mathrm{bar}}(I / N, I)$,

$$
\tau_{n}^{\prime}\left(x_{0}, x_{1}, \ldots, x_{n}\right)=\left(x_{0}, \tau\left(x_{1}\right), \ldots, \tau\left(x_{n}\right)\right),
$$

for all $x_{0}, \ldots, x_{n} \in I$.

Lemma 3.3. If $I$ is $H$-unital algebra, then $\tau_{*}^{\prime}: C_{*}^{\mathrm{bar}}(I) \rightarrow C_{*}^{\mathrm{bar}}(I / N, I)$ is a quasiisomorphism.

Proof. Since this lemma can be proved essentially in the same way as the previous one, we omit its proof.

Let $\gamma: I \rightarrow A$ be a crossed module of algebras. The Peiffer Identity implies Ker $\gamma \subset$ $\operatorname{Ann}(I)$. Set $N=\operatorname{Ker} \gamma$. Since $\mathbf{k}$ is a field, $C_{*}(I / N, I)$ can be considered as subcomplex of $C_{*}(A, I)$.

Let $X \subset I / N$ be a basis of $I / N$ over $\mathbf{k}$ and $Y \subset A \backslash(I / N)$ a basis of $A /(I / N)$ over k. Assume that $x_{0} \otimes x_{1} \otimes \cdots \otimes x_{n} \in I \otimes A^{\otimes n}$ is such that $x_{i} \in X \cup Y$ for all $i$, $i \in\{1, \ldots, n\}, x_{0} \in I$ and $x_{0} \neq 0$. We introduce a notation $l:=l\left(x_{0} \otimes x_{1} \otimes \cdots \otimes x_{n}\right)$ defined as follows: $l=0$ if $x_{i} \in Y$ for all $i, i \in\{1, \ldots, n\}$, and if there exists $i \geq 1$ such that $x_{i} \in X$ then $l=\max \left\{i \mid x_{1}, \ldots, x_{i} \in X\right.$ and $\left.x_{i+1} \in Y\right\}$.

For any non trivial $w \in C_{n}(A, I)$ we have

$$
w=\sum_{j=1}^{m} x_{0 j} \otimes x_{1 j} \otimes \cdots \otimes x_{n j},
$$

where $x_{0 j} \in I, x_{0 j} \neq 0, x_{i j} \in X \cup Y$ and

$$
\left(x_{1 j}, x_{2 j}, \ldots, x_{n j}\right) \neq\left(x_{1 j^{\prime}}, x_{2 j^{\prime}}, \ldots, x_{n j^{\prime}}\right), \quad j \neq j^{\prime} .
$$

Define $l(w)$ as follows:

$$
l(w)=\min _{j}\left\{l\left(x_{0 j} \otimes x_{1 j} \otimes \cdots \otimes x_{n j}\right)\right\} .
$$

Clearly if $l(w)=n$, then $w \in C_{n}(I / N, I)$. We need the following lemma.

Lemma 3.4. If $w \in C_{n}(A, I)$ is such that $b(w) \in C_{n-1}(I / N, I)$ and $l(w)<n$, then either $w \in \operatorname{Im} b$ or there exists $w^{\prime} \in C_{n}(A, I)$ such that $l\left(w^{\prime}\right)>l(w)$ and $w-w^{\prime} \in \operatorname{Im} b$.

Proof. Without loss of generality, we can assume that

$$
w=\sum_{j=1}^{m_{1}} x_{0 j} \otimes x_{1 j} \otimes \cdots \otimes x_{n j}+\sum_{j=m_{1}+1}^{m} x_{0 j} \otimes x_{1 j} \otimes \cdots \otimes x_{n j}=w_{1}+w_{2},
$$

where $l(w)=l\left(x_{0 j} \otimes x_{1 j} \otimes \cdots \otimes x_{n j}\right)$ when $1 \leq j \leq m_{1}$ and $l(w)<l\left(x_{0 j} \otimes x_{1 j} \otimes \cdots \otimes x_{n j}\right)$ when $j>m_{1}$. One has:

$$
w_{1}=\sum_{j=1}^{m_{1}} x_{0 j} \otimes x_{1 j} \otimes \cdots \otimes x_{n j}=\sum_{s=1}^{m_{2}} w_{0 s} \otimes x_{l+1 s} \otimes \cdots \otimes x_{n s},
$$

where $w_{0 s} \in I \otimes(I / N)^{\otimes l}, l=l(w)$ and $\left(x_{l+1} s, \ldots, x_{n s}\right) \neq\left(x_{l+1 s^{\prime}}, \ldots, x_{n s^{\prime}}\right)$ when $s \neq s^{\prime}$. Since $b(w) \in C_{n-1}(I / N, I)$, either $b(w)=0$ or $l(b(w))=n-1$. One can verify that

$$
\text { either } \quad b(w)-\sum_{s=1}^{m_{2}} b^{\prime}\left(w_{0 s}\right) \otimes x_{l+1 s} \otimes \cdots \otimes x_{n s}=0 \quad \text { or }
$$




$$
l\left(b(w)-\sum_{s=1}^{m_{2}} b^{\prime}\left(w_{0 s}\right) \otimes x_{l+1 s} \otimes \cdots \otimes x_{n s}\right)>l(w)-1 .
$$

Assume that there exists $s, 1 \leq s \leq m_{2}$, such that $b^{\prime}\left(w_{0 s}\right) \neq 0$. Then

$$
l\left(\sum_{s=1}^{m_{2}} b^{\prime}\left(w_{0 s}\right) \otimes x_{l+1 s} \otimes \cdots \otimes x_{n s}\right)=l(w)-1 .
$$

On the other hand, the following equality

$\sum_{s=1}^{m_{2}} b^{\prime}\left(w_{0 s}\right) \otimes x_{l+1 s} \otimes \cdots \otimes x_{n s}=b(w)-\left(b(w)-\sum_{s=1}^{m_{2}} b^{\prime}\left(w_{0 s}\right) \otimes x_{l+1 s} \otimes \cdots \otimes x_{n s}\right)$,

and (3.1) implies that

$$
l\left(\sum_{s=1}^{m_{2}} b^{\prime}\left(w_{0 s}\right) \otimes x_{l+1 s} \otimes \cdots \otimes x_{n s}\right)>l(w)-1,
$$

that is, a contradiction. Hence $b^{\prime}\left(w_{0 s}\right)=0$ for all $s, 1 \leq s \leq m_{2}$. By Lemma 3.3, there exists $v_{s} \in I \otimes(I / N)^{\otimes l+1}$ such that $b^{\prime}\left(v_{s}\right)=w_{0 s}$. Set

$$
\bar{w}=\sum_{s=1}^{m_{2}} v_{s} \otimes x_{l+1 s} \otimes \cdots \otimes x_{n s} .
$$

Then either $b(\bar{w})=w$ or $l(b(\bar{w})-w)>l(w)$.

Proof of Proposition 2.6. Using Lemma 3.1, it suffices to show that $C_{*}(I / N, I)$ and $C_{*}(A, I)$ are quasi-isomorphic. Let $\widetilde{C}_{*}(A, I)$ be a complex defined from the following short exact sequence:

$$
0 \longrightarrow C_{*}(I / N, I) \longrightarrow C_{*}(A, I) \longrightarrow \widetilde{C}_{*}(A, I) \longrightarrow 0 .
$$

Our aim is to show that $\widetilde{C}(A, I)$ is acyclic. Let $w \in C_{n}(A, I), b(w) \in C_{n-1}(I / N, I)$ and $l(w)<n$. By Lemma 3.4, either $w \in \operatorname{Im} b$ or there exists $w^{\prime} \in C_{n}(A, I)$ such that $l\left(w^{\prime}\right)=n$ and $w-w^{\prime} \in \operatorname{Im} b$. In other words, if $w \in C_{n}(A, I), b(w) \in C_{n-1}(I / N, I)$ and $w \notin C_{n}(I / N, I)$, then either $w \in \operatorname{Im} b$ or there exists $w^{\prime} \in C_{n}(I / N, I)$ such that $w-w^{\prime} \in \operatorname{Im} b$. This means that every cycle of the complex $\widetilde{C}_{*}(A, I)$ is a boundary.

\section{Acknowledgments}

The two authors were supported by Ministerio de Ciencia e Innovación (Spain), Grant MTM2009-14464-C02 (European FEDER support included) and by Xunta de Galicia, Grant Incite09 207215 PR. The first author was also partially supported by Xunta de Galicia, Grant Incite09 207151 PR.

\section{References}

[1] A. Connes, Cohomologie cyclique et foncteurs Ext ${ }^{n}$, C. R. Acad. Sci. Paris Sér. I Math. 296(23) (1983), 953-958.

[2] G. Donadze and M. Ladra, Hochschild homology with coefficients in an H-unital ideal, Homology, Homotopy Appl. 11(2) (2009), 1-4.

[3] G.J. Ellis, Higher-dimensional crossed modules of algebras, J. Pure Appl. Algebra 52(3) (1988), 277-282. 
[4] D. Kaledin, Cyclic homology with coefficients, in Algebra, arithmetic, and geometry: in honor of Yu. I. Manin. Vol. II, Vol. 270 of Progr. Math., 23-47, Birkhäuser Boston Inc., Boston, MA (2009).

[5] J.-L. Loday, Cyclic homology, Vol. 301 of Grundlehren der Mathematischen Wissenschaften [Fundamental Principles of Mathematical Sciences], Springer-Verlag, Berlin (1992). Appendix E by María O. Ronco.

[6] M. Wodzicki, Excision in cyclic homology and in rational algebraic K-theory, Ann. Math. (2) 129(3) (1989), 591-639.

Kerala School of Mathematics, Kunnamangalam P.O., Kozhikode - 673 571, Kerala, INDIA

E-mail address: gdonad@gmail.com

Departamento de Álgebra, Universidad de Santiago de Compostela, 15782 Santiago de Compostela, Spain

E-mail address: manuel.ladra@usc.es 
